TITLE AND AUTHORSHIP

\title{
Determination of MCPD Esters and Glycidyl Esters by Microwave Extraction in different Foodstuffs
}

Corinne Marc*, Valérie Drouard-Pascarel, Cécile Rétho, Patrice Janvion, Frédéric

Saltron

Service Commun des Laboratoires, 25 Avenue de la République ,91744 Massy, France

* corinne.marc@scl.finances.gouv.fr; +33169538751 


\section{ASSOCIATED CONTENT}

Table S1. Comparison of the extraction of fat between the Röse Gottlieb method and the microwave method.

Table S2. Microwave extraction : comparison between tert-butyl methyl ether and ethyl acetate and between stirrings rods oval-shaped and rectangular stirring rods.

Table S3. Results of linearity study for 3-MCPD esters

Table S4. Results of linearity study for 2-MCPD esters

Table S5. Results of linearity study for glycidyl esters

Table S6. Results of checking of $L O Q$ for oils

Table S7. Results of checking of LOQ for crisps and chips

Table S8. Results of checking of LOQ for infant formula

Table S9. Results of accuracy for oils

Table S10. Results of accuracy for crisps and chips

Table S11. Results of accuracy for infant formula

Table S12. Results of recovery for oils

Table S13. Results of recovery for crisps and chips

Table S14. Results of recovery for infant formula

Table S15. Results of inter laboratory tests 
Table S1. Results of the comparison of the extraction of fat between the Röse Gottlieb method and the microwave method.

\begin{tabular}{|c|c|c|c|}
\hline Matrix & $\begin{array}{c}\text { Fat percentage } \\
\text { to package }\end{array}$ & $\begin{array}{c}\text { Fat percentage determined by the } \\
\text { Röse Gottlieb method }\end{array}$ & $\begin{array}{c}\text { Fat percentage } \\
\text { determined by } \\
\text { microwave method }\end{array}$ \\
\hline Liquid infant formula & $3.3 \mathrm{~g} / 100 \mathrm{~mL}$ & $3.3 \mathrm{~g} / 100 \mathrm{~mL}$ & $3.1 \mathrm{~g} / 100 \mathrm{~mL}$ \\
\hline Solid infant formula & $24 \mathrm{~g} / 100 \mathrm{~g}$ & $24.05 \mathrm{~g} / 100 \mathrm{~mL}$ & $23.95 \mathrm{~g} / 100 \mathrm{~mL}$ \\
\hline Crisp & $35 \mathrm{~g} / 100 \mathrm{~g}$ & $33.8 \mathrm{~g} / 100 \mathrm{~g}$ & $33.2 \mathrm{~g} / 100 \mathrm{~g}$ \\
\hline Crisp & $34 \mathrm{~g} / 100 \mathrm{~g}$ & $33.2 \mathrm{~g} / 100 \mathrm{~g}$ & $32.2 \mathrm{~g} / 100 \mathrm{~g}$ \\
\hline Chips & Not mentioned & $13.4 \mathrm{~g} / 100 \mathrm{~g}$ & $12.8 \mathrm{~g} / 100 \mathrm{~g}$ \\
\hline Chips & Not mentioned & $10.7 \mathrm{~g} / 100 \mathrm{~g}$ & $11.2 \mathrm{~g} / 100 \mathrm{~g}$ \\
\hline
\end{tabular}


Table S2. Microwave extraction: comparison between tert-butyl methyl ether and ethyl acetate and between stirrings rods oval-shaped and rectangular stirring rods.

\begin{tabular}{|c|c|c|c|c|c|}
\hline Matrix & Stirring rods & Solvent & $\begin{array}{c}\text { Recovery of 3- } \\
\text { MCPD esters (\%) }\end{array}$ & $\begin{array}{c}\text { Recovery } \\
\text { of 2- } \\
\text { MCPD } \\
\text { esters } \\
(\%)\end{array}$ & $\begin{array}{c}\text { Recovery } \\
\text { of } \\
\text { glycidyl } \\
\text { esters } \\
(\%)\end{array}$ \\
\hline Crisp A & Rectangular & $\begin{array}{c}\text { Tert-butyl methyl } \\
\text { ether }\end{array}$ & 59 & 64 & 55 \\
\hline Crisp A & Rectangular & $\begin{array}{c}\text { Tert-butyl methyl } \\
\text { ether }\end{array}$ & 33 & 36 & 31 \\
\hline Crisp A & Oval-shaped & $\begin{array}{c}\text { Tert-butyl methyl } \\
\text { ether }\end{array}$ & 67 & 55 & 51 \\
\hline Crisp A & Oval-shaped & $\begin{array}{c}\text { Tert-butyl methyl } \\
\text { ether }\end{array}$ & 61 & 58 & 46 \\
\hline Crisp B & Oval-shaped & $\begin{array}{c}\text { Tert-butyl methyl } \\
\text { ether }\end{array}$ & 63 & 61 & 70 \\
\hline Crisp B & Oval-shaped & $\begin{array}{c}\text { Tert-butyl methyl } \\
\text { ether }\end{array}$ & 63 & 59 & 72 \\
\hline Crisp B & Oval-shaped & Ethyl acetate & 82 & 78 & 73 \\
\hline Crisp B & Oval-shaped & Ethyl acetate & 87 & 82 & 80 \\
\hline Liquid infant & Rectangular & Ethyl acetate & Indeed, thanks to the built-in camera, no \\
formula C & agitation was observed in the tube \\
\hline $\begin{array}{c}\text { Liquid infant } \\
\text { formula C }\end{array}$ & Oval-shaped & Ethyl acetate & 76 & 73 & 59 \\
\hline $\begin{array}{c}\text { Liquid infant } \\
\text { formula C }\end{array}$ & Oval-shaped & Tert-butyl methyl \\
ether & $\begin{array}{c}\text { With tert-butyl methyl ether, we observed an } \\
\text { emulsion and the agitation seemed harder. }\end{array}$ \\
\hline
\end{tabular}


Table S3. Results of linearity study for 3-MCPD esters

\begin{tabular}{|c|c|c|c|c|c|c|c|c|c|c|c|}
\hline \multicolumn{12}{|c|}{ 3-MCPD esters } \\
\hline & Lev.1 & Lev. 2 & Lev. 3 & Lev. 4 & Lev. 5 & Lev. 6 & Lev. 7 & Lev. 8 & $y=b x$ & \multirow{2}{*}{$\begin{array}{l}\text { coefficient of } \\
\text { determination }\end{array}$} & \multirow{2}{*}{$\begin{array}{l}\text { Correlation } \\
\text { coefficient }\end{array}$} \\
\hline Theoretical values & 0,062 & 0,126 & 0,25 & 0,502 & 0,752 & 1,255 & 1,755 & 2,257 & $b$ & & \\
\hline \multirow[b]{2}{*}{ Obtained values } & 0,064 & 0,140 & 0,310 & 0,486 & 0,824 & 1,465 & 2,131 & 2,736 & 1,1942 & 0,998505 & 0,999252 \\
\hline & 0,063 & 0,139 & 0,268 & 0,588 & 0,849 & 1,468 & 1,976 & 2,542 & 1,1334 & 0,999767 & 0,999884 \\
\hline \multirow{4}{*}{$\begin{array}{l}\text { Values calculated } \\
\text { from the equation } \\
\text { of the calibration } \\
\text { line }\end{array}$} & 0,04 & 0,10 & 0,24 & 0,41 & 0,71 & 1,22 & 1,77 & 2,31 & & & \\
\hline & 0,05 & 0,12 & 0,26 & 0,41 & 0,69 & 1,23 & 1,78 & 2,29 & & & \\
\hline & 0,06 & 0,12 & 0,24 & 0,52 & 0,75 & 1,30 & 1,74 & 2,24 & & & \\
\hline & 0,05 & 0,12 & 0,25 & 0,41 & 0,68 & 1,23 & 1,77 & 2,30 & & & \\
\hline \multirow{3}{*}{$\begin{array}{l}\text { Relative deviations } \\
\text { between values } \\
\text { calculated from the } \\
\text { equation of the } \\
\text { calibration line and } \\
\text { theorical values }\end{array}$} & $-10,3 \%$ & $-2,7 \%$ & $-5,4 \%$ & $3,3 \%$ & $-0,4 \%$ & $3,2 \%$ & $-0,7 \%$ & $-0,6 \%$ & & & \\
\hline & $-11,6 \%$ & $-4,7 \%$ & $1,9 \%$ & $-17,5 \%$ & $-9,5 \%$ & $-1,8 \%$ & $0,8 \%$ & $2,0 \%$ & & & \\
\hline & $15,2 \%$ & $16,7 \%$ & $8,6 \%$ & $-10,1 \%$ & $-11,4 \%$ & $-3,5 \%$ & $0,4 \%$ & $2,4 \%$ & & & \\
\hline $\begin{array}{c}\text { EMA } \% \text { set by the } \\
\text { laboratory }\end{array}$ & $50 \%$ & $20 \%$ & $20 \%$ & $20 \%$ & $20 \%$ & $20 \%$ & $20 \%$ & $20 \%$ & & & \\
\hline $\begin{array}{c}\text { Conclusion about } \\
\text { the linearity }\end{array}$ & Checked & Checked & Checked & Checked & Checked & Checked & Checked & Checked & & & \\
\hline
\end{tabular}


Table S4. Results of linearity study for 2-MCPD esters

\begin{tabular}{|c|c|c|c|c|c|c|c|c|c|c|c|}
\hline \multicolumn{12}{|c|}{ 2-MCPD esters } \\
\hline & Lev.1 & Lev. 2 & Lev. 3 & Lev. 4 & Lev. 5 & Lev. 6 & Lev.7 & Lev. 8 & $y=b x$ & \multirow{2}{*}{$\begin{array}{l}\text { coefficient of } \\
\text { determination }\end{array}$} & \multirow{2}{*}{$\begin{array}{l}\text { Correlation } \\
\text { coefficient }\end{array}$} \\
\hline Theoretical values & 0,064 & 0,129 & 0,257 & 0,51 & 0,774 & 1,276 & 1,805 & 2,319 & $b$ & & \\
\hline \multirow{5}{*}{ Obtained values } & 0,313 & 0,667 & 1,366 & 2,417 & 3,796 & 7,205 & 9,644 & 13,584 & 5,5960 & 0,996964 & 0,998481 \\
\hline & 0,319 & 0,703 & 1,468 & 2,241 & 3,659 & 6,628 & 9,692 & 12,635 & 5,3270 & 0,998223 & 0,999111 \\
\hline & 0,196 & 0,609 & 1,226 & 2,961 & 4,288 & 7,320 & 10,205 & 12,697 & 5,5701 & 0,999393 & 0,999696 \\
\hline & 0,281 & 0,637 & 1,310 & 2,789 & 4,291 & 7,292 & 10,421 & 13,043 & 5,6683 & 0,999704 & 0,999852 \\
\hline & 0,298 & 0,661 & 1,421 & 2,387 & 3,769 & 7,239 & 10,316 & 13,268 & 5,6405 & 0,998152 & 0,999076 \\
\hline \multirow{5}{*}{$\begin{array}{l}\text { Values calculated } \\
\text { from the equation } \\
\text { of the calibration } \\
\text { line }\end{array}$} & 0,06 & 0,12 & 0,24 & 0,43 & 0,68 & 1,29 & 1,72 & 2,43 & & & \\
\hline & 0,06 & 0,13 & 0,28 & 0,42 & 0,69 & 1,24 & 1,82 & 2,37 & & & \\
\hline & 0,04 & 0,11 & 0,22 & 0,53 & 0,77 & 1,31 & 1,83 & 2,28 & & & \\
\hline & 0,05 & 0,11 & 0,23 & 0,49 & 0,76 & 1,29 & 1,84 & 2,30 & & & \\
\hline & 0,05 & 0,12 & 0,25 & 0,42 & 0,67 & 1,28 & 1,83 & 2,35 & & & \\
\hline \multirow{5}{*}{$\begin{array}{l}\text { Relative deviations } \\
\text { between values } \\
\text { calculated from the } \\
\text { equation of the } \\
\text { calibration line and } \\
\text { theorical values }\end{array}$} & $-12,6 \%$ & $-7,6 \%$ & $-5,0 \%$ & $-15,3 \%$ & $-12,4 \%$ & $0,9 \%$ & $-4,5 \%$ & $4,7 \%$ & & & \\
\hline & $-6,4 \%$ & $2,3 \%$ & $7,2 \%$ & $-17,5 \%$ & $-11,3 \%$ & $-2,5 \%$ & $0,8 \%$ & $2,3 \%$ & & & \\
\hline & $-45,0 \%$ & $-15,2 \%$ & $-14,4 \%$ & $4,2 \%$ & $-0,5 \%$ & $3,0 \%$ & $1,5 \%$ & $-1,7 \%$ & & & \\
\hline & $-22,5 \%$ & $-12,9 \%$ & $-10,1 \%$ & $-3,5 \%$ & $-2,2 \%$ & $0,8 \%$ & $1,9 \%$ & $-0,8 \%$ & & & \\
\hline & $-17,5 \%$ & $-9,2 \%$ & $-2,0 \%$ & $-17,0 \%$ & $-13,7 \%$ & $0,6 \%$ & $1,3 \%$ & $1,4 \%$ & & & \\
\hline $\begin{array}{l}\text { EMA\% set by the } \\
\text { laboratory }\end{array}$ & $50 \%$ & $20 \%$ & $20 \%$ & $20 \%$ & $20 \%$ & $20 \%$ & $20 \%$ & $20 \%$ & & & \\
\hline $\begin{array}{c}\text { Conclusion about } \\
\text { the linearity }\end{array}$ & Checked & Checked & Checked & Checked & Checked & Checked & Checked & Checked & & & \\
\hline
\end{tabular}


Table S5. Results of linearity study for glycidyl esters

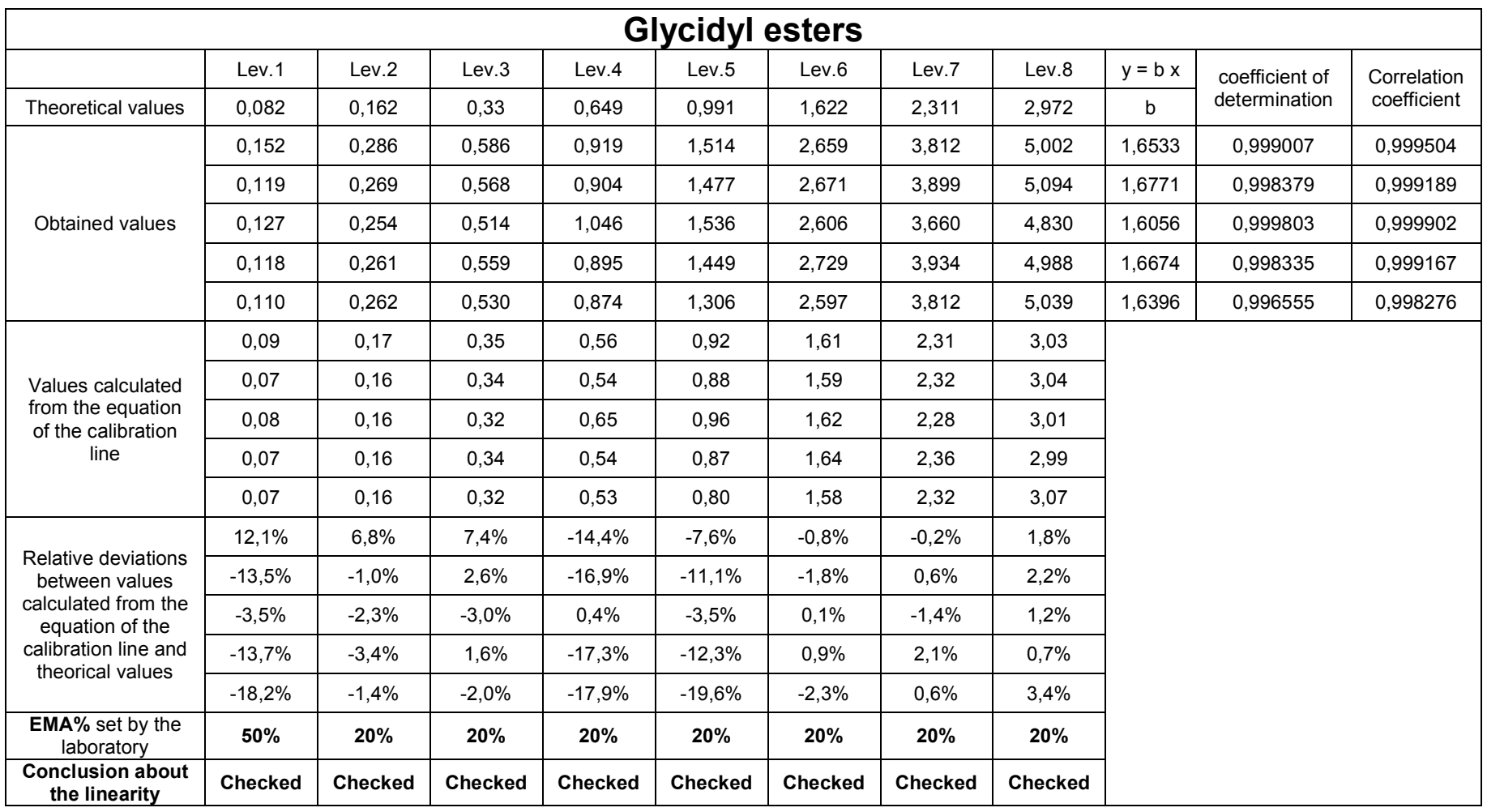


Table S6. Results of checking of LOQ for oils

\begin{tabular}{|c|c|c|c|}
\hline & $\begin{array}{c}\text { 3-MCPD } \\
\text { esters }\end{array}$ & $\begin{array}{c}\text { 2-MCPD } \\
\text { esters }\end{array}$ & $\begin{array}{c}\text { Glycidyl } \\
\text { esters }\end{array}$ \\
\hline LOQ to checking & 0.1 & 0.1 & 0.1 \\
\hline EMA : maximum acceptable deviations & $60 \%$ & $60 \%$ & $60 \%$ \\
\hline Average $: \overline{\bar{x}}$ & 0,10950 & 0,09320 & 0,10980 \\
\hline Standard deviation for intermediate precision : $s_{F I}$ & 0,00754 & 0,01620 & 0,01640 \\
\hline $\begin{array}{l}\text { CV (Coefficient of variation) in intermediate precision in } \\
\%\end{array}$ & $6,9 \%$ & $17,4 \%$ & $14,9 \%$ \\
\hline Absolute bias & 0,0045 & $-0,0148$ & 0,0128 \\
\hline Relative bias in $\%$ & $4,3 \%$ & $-13,7 \%$ & $13,2 \%$ \\
\hline Numbers of series & 5 & 5 & 5 \\
\hline Number of repetitions per series & 2 & 2 & 2 \\
\hline Repeatability variance & 9,30E-06 & $8,30 \mathrm{E}-05$ & 4,54E-05 \\
\hline Variance of means & $5,23 E-05$ & $2,21 \mathrm{E}-04$ & $2,46 \mathrm{E}-04$ \\
\hline Inter-series variance & $4,76 \mathrm{E}-05$ & $1,80 \mathrm{E}-04$ & $2,24 \mathrm{E}-04$ \\
\hline Variance of intermediate precision & 5,69E-05 & $2,63 \mathrm{E}-04$ & $2,69 \mathrm{E}-04$ \\
\hline \multicolumn{4}{|c|}{ Interpretation of the accuracy (NF T90-210) } \\
\hline Reference & 0,1050 & 0,1080 & 0,0970 \\
\hline EMA : maximum acceptable deviations & 0,0630 & 0,0648 & 0,0582 \\
\hline High limit of acceptability & 0,1680 & 0,1728 & 0,1552 \\
\hline$\overline{\bar{x}}+\left(2 * s_{F I}\right)$ & 0,1246 & 0,1256 & 0,1426 \\
\hline$\overline{\bar{x}}-\left(2 * s_{F I}\right)$ & 0,0944 & 0,0608 & 0,0770 \\
\hline Low limit of acceptability & 0,0420 & 0,0432 & 0,0388 \\
\hline Conclusion & $\begin{array}{l}\text { The LOQ is } \\
\text { checked }\end{array}$ & $\begin{array}{l}\text { The LOQ is } \\
\text { checked }\end{array}$ & $\begin{array}{l}\text { The LOQ is } \\
\text { checked }\end{array}$ \\
\hline
\end{tabular}


Table S7. Results of checking of LOQ for crisps and chips

\begin{tabular}{|c|c|c|c|}
\hline & $\begin{array}{c}\text { 3-MCPD } \\
\text { esters }\end{array}$ & $\begin{array}{c}\text { 2-MCPD } \\
\text { esters }\end{array}$ & $\begin{array}{c}\text { Glycidyl } \\
\text { esters }\end{array}$ \\
\hline LOQ to checking & 0.02 & 0.02 & 0.02 \\
\hline EMA : maximum acceptable deviations & $60 \%$ & $60 \%$ & $60 \%$ \\
\hline Average $: \overline{\bar{x}}$ & 0,02150 & 0,01900 & 0,01870 \\
\hline Standard deviation for intermediate precision : $s_{F I}$ & 0,00572 & 0,00436 & 0,00354 \\
\hline $\begin{array}{l}\text { CV (Coefficient of variation) in intermediate precision in } \\
\%\end{array}$ & $26,6 \%$ & $23,0 \%$ & $18,9 \%$ \\
\hline Absolute bias & $-0,0005$ & $-0,0030$ & $-0,0013$ \\
\hline Relative bias in $\%$ & $-2,3 \%$ & $-13,6 \%$ & $-6,5 \%$ \\
\hline Numbers of series & 5 & 5 & 5 \\
\hline Number of repetitions per series & 2 & 2 & 2 \\
\hline Repeatability variance & 1,09E-05 & $3,80 \mathrm{E}-06$ & $1,25 \mathrm{E}-05$ \\
\hline Variance of means & $2,73 \mathrm{E}-05$ & $1,71 \mathrm{E}-05$ & $3,45 \mathrm{E}-06$ \\
\hline Inter-series variance & $2,18 \mathrm{E}-05$ & $1,52 \mathrm{E}-05$ & $0,00 \mathrm{E}+00$ \\
\hline Variance of intermediate precision & $3,27 \mathrm{E}-05$ & 1,90E-05 & $1,25 \mathrm{E}-05$ \\
\hline \multicolumn{4}{|c|}{ Interpretation of the accuracy (NF T90-210) } \\
\hline Reference & 0,0220 & 0,0220 & 0,0200 \\
\hline EMA : maximum acceptable deviations & 0,0132 & 0,0132 & 0,0120 \\
\hline High limit of acceptability & 0,0352 & 0,0352 & 0,0320 \\
\hline$\overline{\bar{x}}+\left(2 * s_{F I}\right)$ & 0,0329 & 0,0277 & 0,0258 \\
\hline$\overline{\bar{x}}-\left(2 * s_{F I}\right)$ & 0,0101 & 0,0103 & 0,0116 \\
\hline Low limit of acceptability & 0,0088 & 0,0088 & 0,0080 \\
\hline Conclusion & $\begin{array}{l}\text { The LOQ is } \\
\text { checked }\end{array}$ & $\begin{array}{l}\text { The LOQ is } \\
\text { checked }\end{array}$ & $\begin{array}{l}\text { The LOQ is } \\
\text { checked }\end{array}$ \\
\hline
\end{tabular}


Table S8. Results of checking of LOQ for infant formula

\begin{tabular}{|c|c|c|c|}
\hline & $\begin{array}{c}\text { 3-MCPD } \\
\text { esters }\end{array}$ & $\begin{array}{c}\text { 2-MCPD } \\
\text { esters }\end{array}$ & $\begin{array}{c}\text { Glycidyl } \\
\text { esters }\end{array}$ \\
\hline LOQ to checking & 0.0027 & 0.0025 & 0.0026 \\
\hline EMA : maximum acceptable deviations & $60 \%$ & $60 \%$ & $60 \%$ \\
\hline Average $: \overline{\bar{x}}$ & 0,00334 & 0,00278 & 0,00240 \\
\hline Standard deviation for intermediate precision : $s_{F I}$ & 0,00042 & 0,00055 & 0,00053 \\
\hline $\begin{array}{l}\text { CV (Coefficient of variation) in intermediate precision in } \\
\%\end{array}$ & $12,5 \%$ & $19,7 \%$ & $22,0 \%$ \\
\hline Absolute bias & 0,0006 & 0,0003 & $-0,0002$ \\
\hline Relative bias in \% & $21,6 \%$ & $10,8 \%$ & $-6,7 \%$ \\
\hline Numbers of series & 5 & 5 & 5 \\
\hline Number of repetitions per series & 2 & 2 & 2 \\
\hline Repeatability variance & $1,00 \mathrm{E}-08$ & $9,00 \mathrm{E}-08$ & $1,66 \mathrm{E}-07$ \\
\hline Variance of means & 1,69E-07 & $2,56 \mathrm{E}-07$ & $1,96 \mathrm{E}-07$ \\
\hline Inter-series variance & 1,64E-07 & $2,11 \mathrm{E}-07$ & $1,13 \mathrm{E}-07$ \\
\hline Variance of intermediate precision & $1,74 \mathrm{E}-07$ & $3,01 \mathrm{E}-07$ & $2,79 \mathrm{E}-07$ \\
\hline \multicolumn{4}{|c|}{ Interpretation of the accuracy (NF T90-210) } \\
\hline Reference & 0,0027 & 0,0025 & 0,0026 \\
\hline EMA : maximum acceptable deviations & 0,0016 & 0,0015 & 0,0015 \\
\hline High limit of acceptability & 0,0044 & 0,0040 & 0,0041 \\
\hline$\overline{\bar{x}}+\left(2 * s_{F I}\right)$ & 0,0042 & 0,0039 & 0,0035 \\
\hline$\overline{\bar{x}}-\left(2 * S_{F I}\right)$ & 0,0025 & 0,0017 & 0,0013 \\
\hline Low limit of acceptability & 0,0011 & 0,0010 & 0,0010 \\
\hline Conclusion & $\begin{array}{l}\text { The LOQ is } \\
\text { checked }\end{array}$ & $\begin{array}{l}\text { The LOQ is } \\
\text { checked }\end{array}$ & $\begin{array}{l}\text { The LOQ is } \\
\text { checked }\end{array}$ \\
\hline
\end{tabular}


Table S9. Results of accuracy for oils

\begin{tabular}{|c|c|c|c|c|c|c|c|c|c|}
\hline \multirow[b]{2}{*}{ Level } & \multicolumn{3}{|c|}{ 3-MCPD esters } & \multicolumn{3}{|c|}{ 2-MCPD esters } & \multicolumn{3}{|c|}{ Glycidyl esters } \\
\hline & 1 & 2 & 3 & 1 & 2 & 3 & 1 & 2 & 3 \\
\hline Reference value & 0,106 & 0,579 & 9,580 & 0,108 & 0,250 & 9,840 & 0,097 & 0,583 & 9,455 \\
\hline $\begin{array}{l}\text { Uncertainty on } \\
\text { reference value }\end{array}$ & 0,005 & 0,022 & 0,479 & 0,005 & 0,013 & 0,492 & 0,005 & 0,018 & 0,473 \\
\hline $\begin{array}{l}\text { EMA : maximum } \\
\text { acceptable deviations } \\
(\%) \quad+\end{array}$ & $60 \%$ & $20 \%$ & $20 \%$ & $60 \%$ & $20 \%$ & $20 \%$ & $60 \%$ & $20 \%$ & $20 \%$ \\
\hline $\begin{array}{l}\text { EMA : maximum } \\
\text { acceptable deviations } \\
(\%)\end{array}$ & $60 \%$ & $20 \%$ & $20 \%$ & $60 \%$ & $20 \%$ & $20 \%$ & $60 \%$ & $20 \%$ & $20 \%$ \\
\hline \multicolumn{10}{|l|}{ Experimental plan } \\
\hline Numbers of series & 5 & 5 & 5 & 5 & 5 & 5 & 5 & 5 & 5 \\
\hline $\begin{array}{l}\text { Number of repetitions } \\
\text { per series }\end{array}$ & 2,00 & 2,00 & 2,00 & 2,00 & 2,00 & 2,00 & 2,00 & 2,00 & 2,00 \\
\hline \multicolumn{10}{|l|}{ Precision } \\
\hline $\begin{array}{l}\text { Repeatability standard } \\
\text { deviation }\end{array}$ & 0,003 & 0,019 & 0,298 & 0,009 & 0,011 & 0,520 & 0,007 & 0,031 & 0,401 \\
\hline Limit of repeatability & 0,009 & 0,053 & 0,834 & 0,026 & 0,030 & 1,455 & 0,019 & 0,087 & 1,123 \\
\hline $\begin{array}{l}\text { Standard deviation for } \\
\text { intermediate precision }\end{array}$ & 0,008 & 0,028 & 0,849 & 0,016 & 0,011 & 0,700 & 0,016 & 0,033 & 0,522 \\
\hline $\begin{array}{l}\text { Intra-laboratory limit of } \\
\text { reproducibility }\end{array}$ & 0,021 & 0,077 & 2,377 & 0,045 & 0,031 & 1,959 & 0,046 & 0,091 & 1,461 \\
\hline $\begin{array}{l}\mathrm{CV} \text { (Coefficient of } \\
\text { variation) of } \\
\text { repeatability }\end{array}$ & $2,79 \%$ & $3,01 \%$ & $3,11 \%$ & $9,78 \%$ & $3,88 \%$ & $5,02 \%$ & $6,14 \%$ & $4,90 \%$ & $4,07 \%$ \\
\hline $\begin{array}{l}\text { CV (Coefficient of } \\
\text { variation) in } \\
\text { intermediate precision }\end{array}$ & $6,89 \%$ & $4,39 \%$ & $8,87 \%$ & $17,39 \%$ & $4,03 \%$ & $6,76 \%$ & $14,94 \%$ & $5,15 \%$ & $5,29 \%$ \\
\hline \multicolumn{10}{|l|}{ Accuracy } \\
\hline Average & 0,110 & 0,628 & 9,568 & 0,093 & 0,276 & 10,346 & 0,110 & 0,634 & 9,858 \\
\hline Bias & 0,004 & 0,049 & $-0,012$ & $-0,015$ & 0,026 & 0,506 & 0,013 & 0,051 & 0,403 \\
\hline $\begin{array}{l}\text { Standardized } \\
\text { difference }\end{array}$ & 0,639 & 1,986 & 0,020 & 1,661 & 1,947 & 0,867 & 1,456 & 2,204 & 0,764 \\
\hline Bias (\%) & $3,79 \%$ & $8,53 \%$ & $-0,13 \%$ & $-13,88 \%$ & $10,48 \%$ & $5,14 \%$ & $13,20 \%$ & $8,77 \%$ & $4,26 \%$ \\
\hline $\begin{array}{l}\text { Conclusion about bias } \\
\text { with NF T90-210 }\end{array}$ & negligible & negligible & negligible & negligible & negligible & negligible & negligible & negligible & negligible \\
\hline \multicolumn{10}{|c|}{ Accuracy study with NF T90-210 } \\
\hline $\begin{array}{l}\text { High limit of } \\
\text { acceptability }\end{array}$ & 0,1688 & 0,6948 & 11,4960 & 0,1732 & 0,3000 & 11,8080 & 0,1552 & 0,6996 & 11,3460 \\
\hline High limit of tolerance & 0,1246 & 0,6836 & 11,2654 & 0,1256 & 0,2985 & 11,7454 & 0,1426 & 0,6994 & 10,9015 \\
\hline Low limit of tolerance & 0,0944 & 0,5732 & 7,8704 & 0,0608 & 0,2539 & 8,9462 & 0,0770 & 0,5688 & 8,8143 \\
\hline $\begin{array}{l}\text { Low limit of } \\
\text { acceptability }\end{array}$ & 0,0422 & 0,4632 & 7,6640 & 0,0433 & 0,2000 & 7,8720 & 0,0388 & 0,4664 & 7,5640 \\
\hline $\begin{array}{l}\text { Conclusion : The } \\
\text { accuracy of method } \\
\text { was }\end{array}$ & Checked & Checked & Checked & Checked & Checked & Checked & Checked & Checked & Checked \\
\hline
\end{tabular}


Table S10. Results of accuracy for crisps and chips

\begin{tabular}{|c|c|c|c|c|c|c|c|c|c|}
\hline \multirow[b]{2}{*}{ Level } & \multicolumn{3}{|c|}{ 3-MCPD esters } & \multicolumn{3}{|c|}{ 2-MCPD esters } & \multicolumn{3}{|c|}{ Glycidyl esters } \\
\hline & 1 & 2 & 3 & 1 & 2 & 3 & 1 & 2 & 3 \\
\hline Reference value & 0,022 & 1,029 & 4,817 & 0,022 & 1,058 & 4,945 & 0,020 & 1,022 & 5,154 \\
\hline $\begin{array}{l}\text { Uncertainty on } \\
\text { reference value }\end{array}$ & 0,001 & 0,051 & 0,241 & 0,001 & 0,053 & 0,247 & 0,001 & 0,051 & 0,258 \\
\hline $\begin{array}{l}\text { EMA : maximum } \\
\text { acceptable deviations } \\
(\%) \quad+\end{array}$ & $60 \%$ & $30 \%$ & $30 \%$ & $60 \%$ & $30 \%$ & $30 \%$ & $60 \%$ & $30 \%$ & $30 \%$ \\
\hline $\begin{array}{l}\text { EMA : maximum } \\
\text { acceptable deviations } \\
(\%)\end{array}$ & $60 \%$ & $30 \%$ & $30 \%$ & $60 \%$ & $30 \%$ & $30 \%$ & $60 \%$ & $30 \%$ & $30 \%$ \\
\hline \multicolumn{10}{|l|}{ Experimental plan } \\
\hline Numbers of series & 5 & 5 & 5 & 5 & 5 & 5 & 5 & 5 & 5 \\
\hline $\begin{array}{l}\text { Number of repetitions } \\
\text { per series }\end{array}$ & 2,00 & 2,00 & 2,00 & 2,00 & 2,00 & 2,00 & 2,00 & 2,00 & 2,00 \\
\hline \multicolumn{10}{|l|}{ Precision } \\
\hline $\begin{array}{l}\text { Repeatability standard } \\
\text { deviation }\end{array}$ & 0,003 & 0,052 & 0,149 & 0,002 & 0,073 & 0,186 & 0,004 & 0,019 & 0,113 \\
\hline Limit of repeatability & 0,009 & 0,146 & 0,418 & 0,005 & 0,203 & 0,519 & 0,010 & 0,054 & 0,316 \\
\hline $\begin{array}{l}\text { Standard deviation for } \\
\text { intermediate precision }\end{array}$ & 0,006 & 0,101 & 0,367 & 0,004 & 0,110 & 0,529 & 0,004 & 0,042 & 0,419 \\
\hline $\begin{array}{l}\text { Intra-laboratory limit of } \\
\text { reproducibility }\end{array}$ & 0,016 & 0,284 & 1,028 & 0,012 & 0,309 & 1,482 & 0,010 & 0,119 & 1,174 \\
\hline $\begin{array}{l}\text { CV (Coefficient of } \\
\text { variation) of } \\
\text { repeatability }\end{array}$ & $15,36 \%$ & $4,67 \%$ & $2,90 \%$ & $10,26 \%$ & $6,45 \%$ & $3,63 \%$ & $18,91 \%$ & $1,69 \%$ & $2,02 \%$ \\
\hline $\begin{array}{l}\text { CV (Coefficient of } \\
\text { variation) in } \\
\text { intermediate precision }\end{array}$ & $26,60 \%$ & $9,07 \%$ & $7,12 \%$ & $22,96 \%$ & $9,80 \%$ & $10,35 \%$ & $18,91 \%$ & $3,71 \%$ & $7,50 \%$ \\
\hline \multicolumn{10}{|l|}{ Accuracy } \\
\hline Average & 0,022 & 1,118 & 5,157 & 0,019 & 1,126 & 5,115 & 0,019 & 1,143 & 5,590 \\
\hline Bias & 0,000 & 0,089 & 0,341 & $-0,003$ & 0,069 & 0,170 & $-0,002$ & 0,120 & 0,436 \\
\hline $\begin{array}{l}\text { Standardized } \\
\text { difference }\end{array}$ & 0,023 & 1,292 & 1,169 & 1,400 & 0,952 & 0,496 & 0,846 & 2,204 & 1,369 \\
\hline Bias (\%) & $-0,30 \%$ & $8,61 \%$ & $7,07 \%$ & $-14,18 \%$ & $6,52 \%$ & $3,43 \%$ & $-7,84 \%$ & $11,75 \%$ & $8,46 \%$ \\
\hline $\begin{array}{l}\text { Conclusion about bias } \\
\text { with NF T90-210 }\end{array}$ & negligible & negligible & negligible & negligible & negligible & negligible & negligible & negligible & negligible \\
\hline \multicolumn{10}{|c|}{ Accuracy study with NF T90-210 } \\
\hline $\begin{array}{l}\text { High limit of } \\
\text { acceptability }\end{array}$ & 0,0345 & 1,3377 & 6,2615 & 0,0354 & 1,3748 & 6,4285 & 0,0325 & 1,3291 & 6,7002 \\
\hline High limit of tolerance & 0,0329 & 1,3204 & 5,8912 & 0,0277 & 1,3472 & 6,1733 & 0,0258 & 1,2273 & 6,4287 \\
\hline Low limit of tolerance & 0,0101 & 0,9148 & 4,4230 & 0,0103 & 0,9056 & 4,0561 & 0,0116 & 1,0577 & 4,7517 \\
\hline $\begin{array}{l}\text { Low limit of } \\
\text { acceptability }\end{array}$ & 0,0086 & 0,7203 & 3,3716 & 0,0089 & 0,7403 & 3,4615 & 0,0081 & 0,7156 & 3,6078 \\
\hline $\begin{array}{l}\text { Conclusion : The } \\
\text { accuracy of method } \\
\text { was }\end{array}$ & Checked & Checked & Checked & Checked & Checked & Checked & Checked & Checked & Checked \\
\hline
\end{tabular}


Table S11. Results of accuracy for infant formula

\begin{tabular}{|c|c|c|c|c|c|c|c|c|c|}
\hline \multirow[b]{2}{*}{ Level } & \multicolumn{3}{|c|}{ 3-MCPD esters } & \multicolumn{3}{|c|}{ 2-MCPD esters } & \multicolumn{3}{|c|}{ Glycidyl esters } \\
\hline & 1 & 2 & 3 & 1 & 2 & 3 & 1 & 2 & 3 \\
\hline Reference value & 0,003 & 0,055 & 0,229 & 0,003 & 0,050 & 0,209 & 0,003 & 0,053 & 0,204 \\
\hline $\begin{array}{l}\text { Uncertainty on } \\
\text { reference value }\end{array}$ & 0,000 & 0,003 & 0,011 & 0,000 & 0,003 & 0,010 & 0,000 & 0,003 & 0,010 \\
\hline $\begin{array}{l}\text { EMA : maximum } \\
\text { acceptable deviations } \\
(\%) \quad+\end{array}$ & $60 \%$ & $30 \%$ & $30 \%$ & $60 \%$ & $30 \%$ & $30 \%$ & $60 \%$ & $30 \%$ & $30 \%$ \\
\hline $\begin{array}{l}\text { EMA : maximum } \\
\text { acceptable deviations } \\
(\%)\end{array}$ & $60 \%$ & $30 \%$ & $30 \%$ & $60 \%$ & $30 \%$ & $30 \%$ & $60 \%$ & $30 \%$ & $30 \%$ \\
\hline \multicolumn{10}{|l|}{ Experimental plan } \\
\hline Numbers of series & 5 & 5 & 5 & 5 & 5 & 5 & 5 & 5 & 5 \\
\hline $\begin{array}{l}\text { Number of repetitions } \\
\text { per series }\end{array}$ & 2,00 & 2,00 & 2,00 & 2,00 & 2,00 & 2,00 & 2,00 & 2,00 & 2,00 \\
\hline \multicolumn{10}{|l|}{ Precision } \\
\hline $\begin{array}{l}\text { Repeatability standard } \\
\text { deviation }\end{array}$ & 0,000 & 0,001 & 0,003 & 0,000 & 0,002 & 0,010 & 0,000 & 0,001 & 0,003 \\
\hline Limit of repeatability & 0,000 & 0,002 & 0,009 & 0,001 & 0,007 & 0,027 & 0,001 & 0,003 & 0,009 \\
\hline $\begin{array}{l}\text { Standard deviation for } \\
\text { intermediate precision }\end{array}$ & 0,000 & 0,002 & 0,009 & 0,001 & 0,005 & 0,023 & 0,001 & 0,002 & 0,008 \\
\hline $\begin{array}{l}\text { Intra-laboratory limit of } \\
\text { reproducibility }\end{array}$ & 0,001 & 0,006 & 0,024 & 0,002 & 0,013 & 0,063 & 0,001 & 0,006 & 0,022 \\
\hline $\begin{array}{l}\text { CV (Coefficient of } \\
\text { variation) of } \\
\text { repeatability }\end{array}$ & $2,99 \%$ & $1,27 \%$ & $1,31 \%$ & $10,79 \%$ & $4,56 \%$ & $4,44 \%$ & $16,98 \%$ & $1,89 \%$ & $1,38 \%$ \\
\hline $\begin{array}{l}\mathrm{CV} \text { (Coefficient of } \\
\text { variation) in } \\
\text { intermediate precision }\end{array}$ & $12,50 \%$ & $3,53 \%$ & $3,57 \%$ & $19,73 \%$ & $8,43 \%$ & $10,35 \%$ & $22,02 \%$ & $3,44 \%$ & $3,39 \%$ \\
\hline \multicolumn{10}{|l|}{ Accuracy } \\
\hline Average & 0,003 & 0,064 & 0,244 & 0,003 & 0,055 & 0,218 & 0,002 & 0,062 & 0,230 \\
\hline Bias & 0,001 & 0,009 & 0,015 & 0,000 & 0,004 & 0,009 & 0,000 & 0,009 & 0,026 \\
\hline $\begin{array}{l}\text { Standardized } \\
\text { difference }\end{array}$ & 2,563 & 3,031 & 1,270 & 0,987 & 1,383 & 0,645 & 0,643 & 3,117 & 2,458 \\
\hline Bias (\%) & $21,63 \%$ & $16,14 \%$ & $6,71 \%$ & $10,85 \%$ & $8,95 \%$ & $4,49 \%$ & $-6,72 \%$ & $16,55 \%$ & $12,99 \%$ \\
\hline $\begin{array}{l}\text { Conclusion about bias } \\
\text { with NF T90-210 }\end{array}$ & negligible & negligible & negligible & negligible & negligible & negligible & negligible & negligible & negligible \\
\hline \multicolumn{10}{|c|}{ Accuracy study with NF T90-210 } \\
\hline $\begin{array}{l}\text { High limit of } \\
\text { acceptability }\end{array}$ & 0,0044 & 0,0714 & 0,2974 & 0,0040 & 0,0652 & 0,2717 & 0,0041 & 0,0694 & 0,2651 \\
\hline High limit of tolerance & 0,0042 & 0,0683 & 0,2616 & 0,0039 & 0,0639 & 0,2636 & 0,0035 & 0,0665 & 0,2460 \\
\hline Low limit of tolerance & 0,0025 & 0,0593 & 0,2267 & 0,0017 & 0,0454 & 0,1732 & 0,0013 & 0,0580 & 0,2148 \\
\hline $\begin{array}{l}\text { Low limit of } \\
\text { acceptability }\end{array}$ & 0,0011 & 0,0384 & 0,1602 & 0,0010 & 0,0351 & 0,1463 & 0,0010 & 0,0374 & 0,1427 \\
\hline $\begin{array}{l}\text { Conclusion: The } \\
\text { accuracy of method } \\
\text { was }\end{array}$ & Checked & Checked & Checked & Checked & Checked & Checked & Checked & Checked & Checked \\
\hline
\end{tabular}


Table S12. Results of recovery for oils

\begin{tabular}{|c|c|c|c|c|c|c|c|c|c|}
\hline \multirow{2}{*}{ Level } & \multicolumn{3}{|c|}{ 3-MCPD esters } & \multicolumn{3}{|c|}{ 2-MCPD esters } & \multicolumn{3}{|c|}{ Glycidyl esters } \\
\hline & 1 & 2 & 3 & 1 & 2 & 3 & 1 & 2 & 3 \\
\hline Mean of recovery & 85,7 & 96,5 & 93,9 & 98,4 & 95,7 & 94,1 & 77,6 & 80,7 & 75,3 \\
\hline $\begin{array}{l}\text { Standard deviation for } \\
\text { intermediate precision }\end{array}$ & 14,8 & 5,9 & 9,8 & 15,6 & 7,2 & 10,8 & 9,9 & 12,0 & 11,5 \\
\hline $\begin{array}{l}\text { CV (Coefficient of } \\
\text { variation) in } \\
\text { intermediate precision }\end{array}$ & $17,3 \%$ & $6,1 \%$ & $10,5 \%$ & $15,8 \%$ & $7,5 \%$ & $11,4 \%$ & $12,7 \%$ & $14,9 \%$ & $15,3 \%$ \\
\hline \multicolumn{10}{|l|}{ Experimental plan } \\
\hline Numbers of series & 5 & 5 & 5 & 5 & 5 & 5 & 5 & 5 & 5 \\
\hline $\begin{array}{l}\text { Number of repetitions } \\
\text { per series }\end{array}$ & 2,00 & 2,00 & 2,00 & 2,00 & 2,00 & 2,00 & 2,00 & 2,00 & 2,00 \\
\hline \multicolumn{10}{|l|}{ Precision } \\
\hline $\begin{array}{l}\text { Variance of } \\
\text { repeatability }\end{array}$ & 172,300 & 26,100 & 96,500 & 115,800 & 27,300 & 115,700 & 3,400 & 31,100 & 71,500 \\
\hline Variance of means & 133.325 & 21.250 & 32.050 & 184.425 & 38.45 & 28.300 & 95.675 & 128.075 & 96.575 \\
\hline Variance inter-series & 47,175 & 8,200 & 0,000 & 126,525 & 24,800 & 0,000 & 93,975 & 112,525 & 60,825 \\
\hline $\begin{array}{l}\text { Variance in } \\
\text { intermediate precision }\end{array}$ & 219,475 & 34,300 & 96,500 & 242,325 & 52,100 & 115,700 & 97,375 & 143,625 & 132,325 \\
\hline $\begin{array}{l}\text { Repeatability standard } \\
\text { deviation }\end{array}$ & 13,1 & 5,1 & 9,8 & 10,8 & 5,2 & 10,8 & 1,8 & 5,6 & 8,5 \\
\hline $\begin{array}{l}\text { CV (Coefficient of } \\
\text { variation) of } \\
\text { repeatability }\end{array}$ & $15,3 \%$ & $5,3 \%$ & $10,5 \%$ & $10,9 \%$ & $5,5 \%$ & $11,4 \%$ & $2,4 \%$ & $6,9 \%$ & $11,2 \%$ \\
\hline
\end{tabular}


Table S13. Results of recovery for crisps and chips

\begin{tabular}{|c|c|c|c|c|c|c|c|c|c|}
\hline & \multicolumn{3}{|c|}{ 3-MCPD esters } & \multicolumn{3}{|c|}{ 2-MCPD esters } & \multicolumn{3}{|c|}{ Glycidyl esters } \\
\hline Level & 1 & 2 & 3 & 1 & 2 & 3 & 1 & 2 & 3 \\
\hline Mean of recovery & 67,5 & 68,2 & 73,8 & 66,6 & 67,9 & 73,6 & 57,4 & 62,0 & 61,2 \\
\hline $\begin{array}{l}\text { Standard deviation for } \\
\text { intermediate precision }\end{array}$ & 8,1 & 6,7 & 9,4 & 12,3 & 9,0 & 13,9 & 7,3 & 6,2 & 6,4 \\
\hline $\begin{array}{l}\mathrm{CV} \text { (Coefficient of } \\
\text { variation) in } \\
\text { intermediate precision }\end{array}$ & $12,1 \%$ & $9,8 \%$ & $12,7 \%$ & $18,4 \%$ & $13,2 \%$ & $18,8 \%$ & $12,7 \%$ & $9,9 \%$ & $10,5 \%$ \\
\hline \multicolumn{10}{|l|}{ Experimental plan } \\
\hline Numbers of series & 5 & 5 & 5 & 5 & 5 & 5 & 5 & 5 & 5 \\
\hline $\begin{array}{l}\text { Number of repetitions } \\
\text { per series }\end{array}$ & 2,00 & 2,00 & 2,00 & 2,00 & 2,00 & 2,00 & 2,00 & 2,00 & 2,00 \\
\hline \multicolumn{10}{|l|}{ Precision } \\
\hline $\begin{array}{l}\text { Variance of } \\
\text { repeatability }\end{array}$ & 20,500 & 45,000 & 10,400 & 14,800 & 51,900 & 12,800 & 5,000 & 38,000 & 12,200 \\
\hline Variance of means & 56.000 & 21.825 & 83.2 & 143.050 & 54.675 & 186.050 & 50.675 & 16.250 & 35.075 \\
\hline Variance inter-series & 45,750 & 0,000 & 78,000 & 135,650 & 28,725 & 179,650 & 48,175 & 0,000 & 28,975 \\
\hline $\begin{array}{l}\text { Variance in } \\
\text { intermediate precision }\end{array}$ & 66,250 & 45,000 & 88,400 & 150,450 & 80,625 & 192,450 & 53,175 & 38,000 & 41,175 \\
\hline $\begin{array}{l}\text { Repeatability standard } \\
\text { deviation }\end{array}$ & 4,5 & 6,7 & 3,2 & 3,8 & 7,2 & 3,6 & 2,2 & 6,2 & 3,5 \\
\hline $\begin{array}{l}\text { CV (Coefficient of } \\
\text { variation) of } \\
\text { repeatability }\end{array}$ & $6,7 \%$ & $9,8 \%$ & $4,4 \%$ & $5,8 \%$ & $10,6 \%$ & $4,9 \%$ & $3,9 \%$ & $9,9 \%$ & $5,7 \%$ \\
\hline
\end{tabular}


Table S14. Results of recovery for infant formula

\begin{tabular}{|c|c|c|c|c|c|c|c|c|c|}
\hline & \multicolumn{3}{|c|}{ 3-MCPD esters } & \multicolumn{3}{|c|}{ 2-MCPD esters } & \multicolumn{3}{|c|}{ Glycidyl esters } \\
\hline Level & 1 & 2 & 3 & 1 & 2 & 3 & 1 & 2 & 3 \\
\hline Mean of recovery & 78,8 & 73,1 & 77,9 & 76,2 & 69,2 & 73,2 & 66,3 & 64,2 & 62,9 \\
\hline $\begin{array}{l}\text { Standard deviation for } \\
\text { intermediate precision }\end{array}$ & 9,3 & 14,2 & 14,8 & 11,0 & 10,3 & 12,4 & 9,7 & 12,3 & 12,4 \\
\hline $\begin{array}{l}\text { CV (Coefficient of } \\
\text { variation) in } \\
\text { intermediate precision }\end{array}$ & $11,8 \%$ & $19,5 \%$ & $19,0 \%$ & $14,4 \%$ & $14,9 \%$ & $17,0 \%$ & $14,6 \%$ & $19,1 \%$ & $19,7 \%$ \\
\hline \multicolumn{10}{|l|}{ Experimental plan } \\
\hline Numbers of series & 5 & 5 & 5 & 5 & 5 & 5 & 5 & 5 & 5 \\
\hline $\begin{array}{l}\text { Number of repetitions } \\
\text { per series }\end{array}$ & 2,00 & 2,00 & 2,00 & 2,00 & 2,00 & 2,00 & 2,00 & 2,00 & 2,00 \\
\hline \multicolumn{10}{|l|}{ Precision } \\
\hline $\begin{array}{l}\text { Variance of } \\
\text { repeatability }\end{array}$ & 47,800 & 31,900 & 34,900 & 46,600 & 44,600 & 19,800 & 53,100 & 112,600 & 96,700 \\
\hline Variance of means & 63.075 & 186.925 & 202.050 & 97.075 & 84.325 & 144.575 & 66.825 & 94.075 & 104.675 \\
\hline Variance inter-series & 39,175 & 170,975 & 184,600 & 73,775 & 62,025 & 134,675 & 40,275 & 37,775 & 56,325 \\
\hline $\begin{array}{l}\text { Variance in } \\
\text { intermediate precision }\end{array}$ & 86,975 & 202,875 & 219,500 & 120,375 & 106,625 & 154,475 & 93,375 & 150,375 & 153,025 \\
\hline $\begin{array}{l}\text { Repeatability standard } \\
\text { deviation }\end{array}$ & 6,9 & 5,6 & 5,9 & 6,8 & 6,7 & 4,4 & 7,3 & 10,6 & 9,8 \\
\hline $\begin{array}{l}\mathrm{CV} \text { (Coefficient of } \\
\text { variation) of } \\
\text { repeatability }\end{array}$ & $8,8 \%$ & $7,7 \%$ & $7,6 \%$ & $9,0 \%$ & $9,7 \%$ & $6,1 \%$ & $11,0 \%$ & $16,5 \%$ & $15,6 \%$ \\
\hline
\end{tabular}


Table S15. Results of inter laboratory tests

\begin{tabular}{|c|c|c|c|c|c|c|c|c|c|}
\hline & \multirow{2}{*}{$\begin{array}{c}\text { Supplier of } \\
\text { inter } \\
\text { laboratory } \\
\text { tests }\end{array}$} & \multirow{2}{*}{$\begin{array}{c}\text { Reference } \\
\text { of inter } \\
\text { laboratory } \\
\text { tests }\end{array}$} & \multirow[b]{2}{*}{ Matrix } & \multicolumn{4}{|c|}{ Statistics of laboratory } & \multicolumn{2}{|c|}{$\begin{array}{c}\text { Statistics of our } \\
\text { laboratory }\end{array}$} \\
\hline & & & & $\begin{array}{l}\text { Reference } \\
\text { valeur }\end{array}$ & $\begin{array}{c}\text { Uncertainty } \\
\text { on } \\
\text { reference } \\
\text { value }\end{array}$ & $\begin{array}{c}\text { Inter } \\
\text { laboratory } \\
\text { standard } \\
\text { deviation }\end{array}$ & $\begin{array}{c}\text { Inter } \\
\text { laboratory } \\
\text { CV (\%) }\end{array}$ & $\begin{array}{c}\text { Value } \\
\text { found by } \\
\text { the } \\
\text { laboratory }\end{array}$ & $\begin{array}{l}\text { Z- } \\
\text { score }\end{array}$ \\
\hline \multirow{13}{*}{ 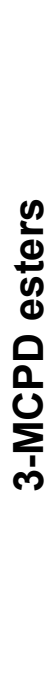 } & \multirow{11}{*}{$\begin{array}{c}\text { aliquots of } \\
\text { the AOCS } \\
\text { Collaborativ } \\
\text { e study (Cd } \\
30-15 \\
\text { coupled with } \\
\text { Cd 29a-13) }\end{array}$} & AOCS-1 & Butter / Margarine & 1,08 & \multirow{11}{*}{$\begin{array}{c}\text { Not } \\
\text { provided }\end{array}$} & 0,15 & $13,9 \%$ & 1,28 & 1,33 \\
\hline & & AOCS-2 & Mayonnaise & 0,16 & & 0,05 & $31,3 \%$ & 0,20 & 0,82 \\
\hline & & AOCS-3 & Butter / Margarine & 0,33 & & 0,13 & $39,4 \%$ & 0,31 & $-0,13$ \\
\hline & & AOCS-4 & Mayonnaise & 0,61 & & 0,13 & $21,3 \%$ & 0,62 & 0,11 \\
\hline & & AOCS-5 & Mayonnaise & 0,12 & & 0,02 & $16,7 \%$ & 0,15 & 1,25 \\
\hline & & AOCS-6 & Mayonnaise & 0,46 & & 0,08 & $17,4 \%$ & 0,59 & 1,56 \\
\hline & & AOCS-7 & Butter / Margarine & 1,08 & & 0,11 & $10,2 \%$ & 1,24 & 1,42 \\
\hline & & AOCS-8 & Mayonnaise & 0,12 & & 0,02 & $16,7 \%$ & 0,15 & 1,70 \\
\hline & & AOCS-9 & Mayonnaise & 0,44 & & 0,06 & $13,6 \%$ & 0,52 & 1,27 \\
\hline & & AOCS-10 & Butter / Margarine & 1,06 & & 0,08 & $7,5 \%$ & 1,22 & 2,00 \\
\hline & & AOCS-11 & Butter / Margarine & 0,25 & & 0,03 & $12,0 \%$ & 0,31 & 1,87 \\
\hline & \multirow{2}{*}{ FAPAS } & Test 2642 & Vegetable oil & 0,58 & 0,02 & 0,12 & $20,0 \%$ & 0,55 & $-0,16$ \\
\hline & & Test 2646 & Vegetable oil & 0,59 & 0,02 & 0,12 & $20,0 \%$ & 0,63 & $-0,16$ \\
\hline \multirow{11}{*}{ 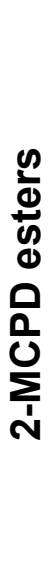 } & \multirow{11}{*}{$\begin{array}{c}\text { aliquots of } \\
\text { the AOCS } \\
\text { Collaborativ } \\
\text { e study (Cd } \\
30-15 \\
\text { coupled with } \\
\text { Cd 29a-13) }\end{array}$} & AOCS-1 & Butter / Margarine & 0,59 & \multirow{11}{*}{$\begin{array}{c}\text { Not } \\
\text { provided }\end{array}$} & 0,15 & $25,4 \%$ & 0,64 & 0,36 \\
\hline & & AOCS-2 & Mayonnaise & 0,08 & & 0,04 & $50,0 \%$ & 0,07 & $-0,18$ \\
\hline & & AOCS-3 & Butter / Margarine & 0,18 & & 0,10 & $55,6 \%$ & 0,14 & $-0,38$ \\
\hline & & AOCS-4 & Mayonnaise & 0,33 & & 0,08 & $24,2 \%$ & 0,30 & $-0,39$ \\
\hline & & AOCS-5 & Mayonnaise & 0,06 & & 0,03 & $50,0 \%$ & 0,07 & 0,20 \\
\hline & & AOCS-6 & Mayonnaise & 0,24 & & 0,07 & $29,2 \%$ & 0,28 & 0,57 \\
\hline & & AOCS-7 & Butter / Margarine & 0,52 & & 0,13 & $25,0 \%$ & 0,55 & 0,20 \\
\hline & & AOCS-8 & Mayonnaise & 0,06 & & 0,03 & $43,3 \%$ & 0,07 & 0,54 \\
\hline & & AOCS-9 & Mayonnaise & 0,21 & & 0,07 & $33,3 \%$ & 0,23 & 0,31 \\
\hline & & AOCS-10 & Butter / Margarine & 0,51 & & 0,10 & $19,6 \%$ & 0,53 & 0,21 \\
\hline & & AOCS-11 & Butter / Margarine & 0,13 & & 0,06 & $46,2 \%$ & 0,13 & 0,07 \\
\hline \multirow{13}{*}{$\begin{array}{l}\frac{0}{0} \\
\frac{1}{0} \\
\frac{0}{0} \\
\frac{2}{0} \\
\frac{0}{0} \\
\frac{2}{0}\end{array}$} & \multirow{11}{*}{$\begin{array}{c}\text { aliquots of } \\
\text { the AOCS } \\
\text { Collaborativ } \\
\text { e study (Cd } \\
30-15 \\
\text { coupled with } \\
\text { Cd 29a-13) }\end{array}$} & AOCS-1 & Butter / Margarine & 2,97 & \multirow{11}{*}{$\begin{array}{c}\text { Not } \\
\text { provided }\end{array}$} & 0,87 & $29,3 \%$ & 3,60 & 0,72 \\
\hline & & AOCS-2 & Mayonnaise & 0,22 & & 0,06 & $27,3 \%$ & 0,22 & $-0,07$ \\
\hline & & AOCS-3 & Butter / Margarine & 0,14 & & 0,08 & $57,1 \%$ & 0,09 & $-0,66$ \\
\hline & & AOCS-4 & Mayonnaise & 0,18 & & 0,07 & $38,9 \%$ & 0,16 & $-0,27$ \\
\hline & & AOCS-5 & Mayonnaise & 0,04 & & 0,04 & $100,0 \%$ & 0,02 & $-0,60$ \\
\hline & & AOCS-6 & Mayonnaise & 0,46 & & 0,14 & $30,4 \%$ & 0,44 & $-0,14$ \\
\hline & & AOCS-7 & Butter / Margarine & 3,01 & & 0,24 & $8,0 \%$ & 3,24 & 0,95 \\
\hline & & AOCS-8 & Mayonnaise & 0,05 & & 0,05 & $100,0 \%$ & 0,02 & $-0,68$ \\
\hline & & AOCS-9 & Mayonnaise & 0,23 & & 0,08 & $34,8 \%$ & 0,22 & $-0,16$ \\
\hline & & AOCS-10 & Butter / Margarine & 2,96 & & 0,32 & $10,8 \%$ & 3,25 & 0,92 \\
\hline & & AOCS-11 & Butter / Margarine & 0,16 & & 0,05 & $31,3 \%$ & 0,16 & 0,00 \\
\hline & \multirow{2}{*}{ FAPAS } & Test 2642 & Vegetable oil & 0,58 & 0,02 & 0,12 & $20,1 \%$ & 0.62 & 0.27 \\
\hline & & Test 2646 & Vegetable oil & 0,26 & 0,01 & 0,05 & $20,0 \%$ & 0,24 & 0,25 \\
\hline
\end{tabular}

